\title{
Primordial Magnetic Fields, Anomalous Matter-Antimatter Fluctuations, and Big Bang Nucleosynthesis
}

\author{
M. Giovannini ${ }^{2}$ and M. E. Shaposhnikov ${ }^{1}$ \\ ${ }^{1}$ CERN, Theory Division, CH-1211 Geneva 23, Switzerland \\ ${ }^{2}$ DAMTP, Silver Street, CB3 9EW Cambridge, United Kingdom
}

(Received 11 August 1997)

\begin{abstract}
We show that the presence of primordial stochastic (hypercharge) magnetic fields before the electroweak phase transition (EWPT) induces baryon-antibaryon inhomogeneities. Depending on the magnetic field spectrum and on the particle physics parameters (such as the strength of the EWPT and electron Yukawa couplings), these fluctuations may survive until the big bang nucleosynthesis (BBN). Their length scale and magnitude can be so large that sizable antimatter domains are present. This provides the possibility of a new type of initial conditions for nonhomogeneous BBN or stringent bounds on primordial magnetic fields. [S0031-9007(97)04920-X]

PACS numbers: 98.80.Cq, 98.62.En
\end{abstract}

Large scale magnetic fields in diffuse astrophysical plasmas represent a well established observational fact for a few decades. It has been realized through the years that magnetic fields coherent over diverse length scales at different epochs may have a variety of quite interesting phenomenological consequences. Magnetic fields coherent today over scales of the order of $30 \mathrm{kpc}$ are measured [1] and have an important role in the dynamics of the galaxy, for example, in confining cosmic rays [2]. Magnetic fields at the nucleosynthesis epoch, even if not directly observable, could change the reaction and the expansion rate at that time.

There are neither compelling theoretical arguments nor motivated phenomenological constraints which could exclude the existence of magnetic fields prior to the nucleosynthesis epoch. Moreover, to explain the origin of the galactic magnetic fields some authors often invoke the dynamo mechanism which might amplify the primordial "seed" magnetic field. It is a challenge to produce large scale seeds, and different ideas were aimed at this purpose. The energy scales involved vary from $\sim 100 \mathrm{MeV}$ for the QCD phase transition [4] to $\sim 100 \mathrm{GeV}$ in the case of the electroweak (EW) physics [5,6] and even closer to the Planck energy scale for inflation or string cosmology $[7,8]$.

The purpose of this Letter is the study of the implications of the primordial magnetic fields which existed even before the EW scale (i.e., for temperatures $\gtrsim 100 \mathrm{GeV}$ ). The origin of these seeds is not essential for us, and consequently we simply assume that they were generated by some mechanism before the EW phase transition. Our main point is that these fields produce baryon and lepton number inhomogeneities (isocurvature fluctuations), which could have an impact on the standard BBN.

Let us start from some qualitative considerations. A unique property of "unbroken" U(1) gauge interaction is the absence of mass of its corresponding vector particle. Static "magnetic" fields are never screened (in the absence of monopoles) and thus homogeneous fields can survive in the plasma for infinite time. Under normal conditions (i.e., small temperatures and small densities of the different fermionic charges) the $\mathrm{SU}(2) \times \mathrm{U}(1)_{Y}$ symmetry is "broken" down to $\mathrm{U}(1)_{\mathrm{EM}}$, the massless field corresponding to $\mathrm{U}(1)_{\mathrm{EM}}$ is the ordinary photon, and the only long-lived field in the plasma is the ordinary magnetic one. At sufficiently high temperatures $T>T_{c}$, the $\mathrm{SU}(2) \times \mathrm{U}(1)_{Y}$ symmetry is "restored," and nonscreened vector modes $Y_{\mu}$ correspond to the $\mathrm{U}(1)_{Y}$ hypercharge group. Hence, if primordial fields existed at $T>T_{c}$, they did correspond to hypercharge rather than $\mathrm{U}(1)_{\mathrm{EM}}$. There are essential differences between the interactions of magnetic fields and the ones of hypermagnetic fields with matter. The ordinary electromagnetic field has a vectorlike coupling to the fermions, while the coupling of the hypercharge fields is chiral. Thus, if hyperelectric $\left(\overrightarrow{\mathcal{E}}_{Y}\right)$ and hypermagnetic $\left(\overrightarrow{\mathcal{H}}_{Y}\right)$ fields are present simultaneously, they cause a variation of the fermionic number according to the anomaly equation, $\partial_{\mu} j_{\mu} \sim\left(g^{\prime 2} / 4 \pi^{2}\right) \overrightarrow{\mathcal{H}}_{Y} \cdot \overrightarrow{\mathcal{E}}_{Y}$ (here $g^{\prime}$ is the hypercharge gauge coupling constant). Now, the presence of nonhomogeneous hypermagnetic fields in the EW plasma with (hyper-) conductivity $\sigma_{c}$ always implies the existence of a related electric field, $\overrightarrow{\mathcal{E}}_{Y} \sim\left(1 / \sigma_{c}\right) \vec{\nabla} \times$ $\overrightarrow{\mathcal{H}}_{Y}$. Since for a general stochastic magnetic background $\left\langle\left(\overrightarrow{\mathcal{H}}_{Y} \cdot \vec{\nabla} \times \overrightarrow{\mathcal{H}}_{Y}\right)^{2}\right\rangle \neq 0$, the nonuniform hypermagnetic field must produce baryon and lepton density perturbations because of the anomaly equation. In what follows we compute the amplitude of isocurvature fluctuations induced by this mechanism and discuss their physical relevance.

The starting point of our discussion will be the generalization of the magnetohydrodynamics (MHD) equations (valid for ordinary electromagnetic plasmas) to the case of hypermagnetic fields with anomalous coupling to the fermionic degrees of freedom (see also [6]). These equations have to be used for $T>T_{c}$. We are interested in a slow dynamics, and we then assume that most of the particle reactions are in thermal equilibrium in the expanding Universe (the list of those include all perturbative strong and weak processes, strong and EW sphalerons, 
Yukawa interactions of $\mu, \tau$, and $s, c, b, t$ quarks). The particle physics processes crucial for our purposes are those related to the $\mathrm{U}(1)_{Y}$ anomaly and to the slowest perturbative reactions with right electron chirality flip (e.g., $e_{R}+$ Higgs $\left.\rightarrow e_{L}+W\right)$. Thus, our variables are the space-dependent hypermagnetic and electric fields $\overrightarrow{\mathcal{H}}_{Y}$, $\overrightarrow{\mathcal{E}}_{Y}$, and right electron chemical potential $\mu_{R}(\vec{x})$. The generalized Maxwell equations in a Friedmann-RobertsonWalker metric with scale factor $a(\tau)$ are

$$
\begin{aligned}
\frac{\partial \vec{H}_{Y}}{\partial \tau} & =-\vec{\nabla} \times \vec{E}_{Y}, \\
\frac{\partial \vec{E}_{Y}}{\partial \tau}+\vec{J}_{Y} & =4 \mu a \vec{H}_{Y}+\vec{\nabla} \times \vec{H}_{Y}, \\
\vec{\nabla} \cdot \vec{H}_{Y} & =0, \quad \vec{\nabla} \cdot \vec{E}_{Y}=0, \\
\vec{J}_{Y} & =\sigma \vec{E}_{Y}, \quad a(\tau) d \tau=d t
\end{aligned}
$$

$\left(\vec{E}_{Y}=a^{2} \overrightarrow{\mathcal{E}}_{Y} ; \vec{H}_{Y}=a^{2} \overrightarrow{\mathcal{H}}_{Y} ; \vec{J}_{Y}=a^{3} \vec{j}_{Y} ; \sigma=\sigma_{c} a\right)$. A new term, proportional to the right electron chemical potential, comes from the anomaly contribution to the effective Lagrangian of hypercharge gauge fields [9],

$$
\begin{gathered}
\delta \mathcal{L}_{Y, e_{R}}=\mu \epsilon_{i j k} Y^{i j} Y^{k}, \quad \mu=\frac{g^{\prime 2}}{4 \pi^{2}} \mu_{R}, \\
\left.Y_{\alpha \beta}=\partial_{[\alpha} Y_{\beta}\right] .
\end{gathered}
$$

Since the EW plasma conductivity is large, $\sigma_{c} \sim \sigma_{0} T$ with $\sigma_{0} \simeq 70-100$ [10], the time derivatives of the electric fields in Eq. (1) can be neglected (in the MHD context this is known as "resistive" approximation [11]). This observation allows one to express the induced electric field in terms of the magnetic one,

$$
\sigma_{c} \overrightarrow{\mathcal{E}}_{Y}=\vec{j}_{Y} \simeq\left(4 \mu \overrightarrow{\mathcal{H}}_{Y}+\vec{\nabla} \times \overrightarrow{\mathcal{H}}_{Y}\right),
$$

and derive an equation for $\overrightarrow{\mathcal{H}}_{Y}$ only. It is interesting to note that the presence of the fermionic chemical potential induces an electric field parallel to the magnetic one.

The set of Eq. (1) has to be supplemented by the kinetic equation for the right electron chemical potential, which accounts for anomalous and perturbative nonconservation of the right electron number,

$$
\begin{aligned}
\frac{\partial}{\partial t}\left(\frac{\mu_{R}}{T}\right)= & -\frac{783}{88} \frac{g^{\prime 2}}{4 \pi^{2} \sigma_{c} T^{3}} \overrightarrow{\mathcal{H}}_{Y} \cdot \vec{\nabla} \times \overrightarrow{\mathcal{H}}_{Y} \\
& -\left(\Gamma+\Gamma_{\mathcal{H}}\right) \frac{\mu_{R}}{T},
\end{aligned}
$$

where $\Gamma$ is the chirality changing rate and

$$
\Gamma_{\mathcal{H}}=\frac{783}{22} \frac{\alpha^{\prime 2}}{\sigma_{c} \pi^{2}} \frac{\left|\mathcal{H}_{Y}\right|^{2}}{T^{2}}, \quad \alpha^{\prime}=\frac{g^{\prime 2}}{4 \pi}
$$

(the numbers 783/88 and 783/22 come from the relationship between $e_{R}$ number density and chemical potential [6]). An interesting consequence of Eqs. (4) and (5) is that in the presence of nonzero uniform magnetic field the right electron number is nonconserved (if $\Gamma=0$ ), even for an Abelian anomaly (cf. Ref. [12]).

Now we are ready to compute baryon number fluctuations produced in our scenario. We notice that at the tem- perature of the EW phase transition $\sim 100 \mathrm{GeV}, \Gamma t \gg 1$. Then, since reactions with right electron chirality flip are in the thermal equilibrium, the adiabatic approximation can be used, and from Eq. (4) we have

$$
\frac{\mu_{R}}{T} \simeq-\frac{783}{88} \frac{\alpha^{\prime}}{\pi \sigma_{c} T^{3}} \frac{\overrightarrow{\mathcal{H}}_{Y} \cdot \vec{\nabla} \times \overrightarrow{\mathcal{H}}_{Y}}{\Gamma+\Gamma_{\mathcal{H}}} .
$$

Clearly, a nonuniform distribution of the right electron chemical potential induces baryon and lepton number perturbations of the same order of magnitude. We are not going to write the explicit formulas since there is an important "storage" effect which amplifies the estimates of Eq. (6) by many orders of magnitude. Equations (3), (4), and (6) imply that

$$
\overrightarrow{\mathcal{H}}_{Y} \cdot \overrightarrow{\mathcal{E}}_{Y} \simeq \frac{\Gamma}{\Gamma+\Gamma \mathcal{H}} \frac{1}{\sigma_{c}} \overrightarrow{\mathcal{H}}_{Y} \cdot \vec{\nabla} \times \overrightarrow{\mathcal{H}}_{Y} \neq 0 .
$$

Now, the change of Abelian Chern-Simons (CS) number is given by the time integral of (7). At the EW phase transition the hypermagnetic fields are converted into ordinary magnetic fields. The latter do not have coupling to the anomaly. Thus the CS number has to be transformed into fermions according to Eq. (4). In order to estimate the produced baryon inhomogeneities at $T=T_{c}$ the full coupled system given by Eqs. (1) and (4) has to be solved for every specific hypermagnetic field configuration [13]. However, if the typical scale of the hypermagnetic field configuration is larger than the hypermagnetic diffusivity scale $\left[L_{\sigma}=(1 / T) \sqrt{M_{0} / \sigma_{c}}\right]$ the hypermagnetic field evolves glued to the EW plasma element as $\left|\overrightarrow{\mathcal{H}}_{Y}\right| \sim 1 / a^{2}$ (i.e., $\left|\overrightarrow{\mathcal{H}}_{Y}\right| \sim$ const). So, the chemical potential of Eq. (6) is approximately constant in time (but not in space). Thus, from Eq. (7) keeping track of the numerical factor we obtain our main result,

$\delta\left(\frac{n_{B}}{s}\right)\left(\vec{x}, t_{c}\right)=\frac{\alpha^{\prime}}{2 \pi \sigma_{c}} \frac{n_{f}}{s} \frac{\overrightarrow{\mathcal{H}}_{Y} \cdot \vec{\nabla} \times \overrightarrow{\mathcal{H}}_{Y}}{\Gamma+\Gamma_{\mathcal{H}}} \frac{\Gamma M_{0}}{T_{c}^{2}}$

$\left[n_{B}\right.$ and $s$ are the baryon and entropy densities, $s=$ $(2 / 45) \pi^{2} N_{\text {eff }} T^{3}, N_{\text {eff }}$ is the effective number of massless degrees of freedom (106.75 for minimal standard model), $\left.M_{0}=M_{p l} / 1.66 \sqrt{N_{\text {eff }}} \simeq 7.1 \times 10^{17} \mathrm{GeV}\right]$. Notice that in Eq. (8) there is an enhancement by a factor $\sim \Gamma M_{0} / T_{c}^{2}$ arising from the time integration of the anomaly term. Some comments are now in order.

(i) For the correctness of Eq. (8) the EW phase transition should be strongly first order. Moreover, a necessary condition for EW baryogenesis [14] must be satisfied. In the opposite case all baryon number fluctuations will be erased by $\mathrm{SU}(2)$ sphalerons as it happens in the minimal standard model (MSM) [15], while this is not necessarily the case for the supersymmetric and other extensions of the standard model [16].

(ii) Besides the primordial hypermagnetic field, an essential quantity which fixes the amplitude of the isocurvature fluctuations is the rate of perturbative right electron chirality flip $\Gamma$. For $\Gamma_{H} \gtrsim \Gamma$ the amplitude of baryon number fluctuations does not depend on the magnitude of 
the magnetic field fluctuations, and it is determined just by their spectral slope. For $\Gamma_{H} \lesssim \Gamma$ the rate of right electron chirality flip cancels out, and the isocurvature fluctuations are fixed both by the magnitude and by the spectral slope of the primordial magnetic fields. In the MSM the rate $\Gamma$ depends crucially upon the electron Yukawa coupling and is known to be $\Gamma=T\left(T_{R} / M_{0}\right)$, where $T_{R} \simeq 80 \mathrm{TeV}$ is the freezing temperature [17] of the right electrons. This number appears to be too small to allow any interesting fluctuations. However, in the extensions of the standard model the rate $\Gamma$ is naturally larger than in MSM. For example, in the MSSM the right electrons Yukawa coupling is larger by a factor $1 / \cos \beta$, which may increase the value of $T_{R}$ by 3 orders of magnitude for experimentally allowed $\tan (\beta) \sim 50$. Cosmologically interesting fluctuations arise at $T_{R}>T^{*} \simeq 10^{5} \mathrm{TeV}$ [13].

We will assume now that $\Gamma \geq \Gamma_{H}$, but similar conclusions hold true for the case $\Gamma \lesssim \Gamma_{H}$ and $T_{R}>T^{*}$ (for details see [13]). In order to compute the amplitude and the spectrum of the baryon number fluctuations we will also suppose that the Fourier modes of the magnetic fields are stochastically distributed, leading to a rotationally and parity invariant two-point function

$$
G_{i j}(r)=\left\langle H_{i}(\vec{x}) H_{j}(\vec{x}+\vec{r})\right\rangle,
$$

where $\langle\ldots\rangle$ denotes an ensemble average. In this case $\left\langle\delta\left(n_{B} / s\right)(\vec{x}, t)\right\rangle=0$ [18], but $\Delta\left(r, t_{c}\right) \neq 0$, where

$$
\Delta\left(r, t_{c}\right)=\sqrt{\left\langle\delta\left(\frac{n_{B}}{s}\right)\left(\vec{x}, t_{c}\right) \delta\left(\frac{n_{B}}{s}\right)\left(\vec{x}+\vec{r}, t_{c}\right)\right\rangle} .
$$

The two-point function of Eq. (9) in Fourier space is:

$$
G_{i j}(k)=k^{2} f(k)\left(\delta_{i j}-k_{i} k_{j} / k^{2}\right) .
$$

For $f(k)$ a power spectrum [modified by the typical exponential decay of small scale magnetic fields given in Eq. (1)] is assumed

$$
\begin{aligned}
f(k) & =\frac{1}{k}\left(\frac{k}{k_{1}}\right)^{-4+\epsilon} \exp \left[-2\left(\frac{k}{k_{\sigma}}\right)^{2}\right], \\
k_{\sigma} & =T \sqrt{\frac{\sigma_{c}}{M_{0}}},
\end{aligned}
$$

where $k_{1}$ characterizes the strength of magnetic fields, and $\epsilon$ is the slope of the spectrum. A physically realistic situation corresponds to the case in which the Green functions of the magnetic hypercharge fields decay at large distance [i.e., $\epsilon>0$ in Eq. (12)]. In $k$ space the magnetic energy density per logarithmic interval of frequency is defined $[7,8]$ as $\rho(k)=d \rho_{H} / d \ln k$ [where $\rho_{H}=\operatorname{Tr} G_{i j}(k)$ ]. Therefore in our case $\rho(k) \sim$ $k^{4}\left(k / k_{1}\right)^{-4+\epsilon}$ which implies that Green's functions decaying at large distances correspond to either "blue" $(\epsilon \geq 0)$ or "violet" $(\epsilon \gg 1)$ logarithmic energy spectra. The case of "red" logarithmic energy spectra $(\epsilon<0)$ will then be left out of our discussion. The case of flat logarithmic energy spectrum $(\epsilon \ll 1)$ may quite naturally appear in string cosmological models [8]. The explicit result for
Eq. (10) at the EW phase transition temperature is

$$
\begin{aligned}
\Delta\left(r, t_{c}\right) & =\frac{45 n_{f} \alpha^{\prime}}{\pi^{2} N_{\mathrm{eff}} \sigma_{0}} \frac{M_{0}}{T_{c}} \frac{\xi^{4-\epsilon} C(\epsilon)}{\left(r T_{c}\right)^{1+\epsilon}}[1+O(\lambda)] \\
C(\epsilon) & =\frac{2^{\epsilon-(3 / 2)} \Gamma\left(\frac{\epsilon}{2}\right)}{\Gamma\left(\frac{3-\epsilon}{2}\right)} \sqrt{\frac{\pi \epsilon(\epsilon+2)}{(3-\epsilon)}} \\
\lambda & \sim\left(\frac{\Gamma}{\Gamma_{H}}\right)^{2}\left(k_{\sigma} r\right)^{-2 \epsilon}
\end{aligned}
$$

[where $\Gamma(z)$ is the Euler gamma function and $\xi=k_{1} / T_{c}$ ]. For a flat spectrum of magnetic fields $(\epsilon \ll 1)$ baryon number fluctuations may be rather large. For example, if the energy sitting in the background magnetic field is comparable with the energy density of the photons, $\left\langle\overrightarrow{\mathcal{H}}_{Y}^{2}\right\rangle \sim T^{4}$ then for the smallest possible scale $\quad r \sim 1 / k_{\sigma} \sim 10^{-9} \times($ EW horizon $\simeq 3 \mathrm{~cm}) \quad$ we get, from Eq. (13), $\delta\left(n_{B} / s\right) \sim\left(\alpha^{\prime} / N_{\text {eff }}\right) \sqrt{M_{0} / \sigma_{c}} \sim 10^{3}$. This number exceeds considerably the measure of the baryon asymmetry of the Universe $n_{B} / s \sim 10^{-10}$, thus small size matter-antimatter domains are possible at the EW scale. At the same time, for even larger scales (possibly relevant for structure formation), the fluctuations of Eq. (13) are quite minute (since their amplitude decreases with the distance as $1 / r^{1+\epsilon}$ ) and may be safely neglected.

We consider now the question whether the fluctuations we found are able to affect the standard BBN. This depends upon the scale of fluctuations at $T=T_{c}$. Short scale fluctuations (well inside the EW horizon) have dissipated by the nucleosynthesis time [19] through the combined action of neutrino inflation and neutron diffusion. Baryon number fluctuations affect BBN provided they are sizable enough over the neutron diffusion scale $\left(3 \times 10^{5} \mathrm{~cm}\right)$ at the onset of nucleosynthesis $\left(T_{\mathrm{NS}} \simeq 100 \mathrm{kev}\right)$ [19]. The neutron diffusion scale, blueshifted to $T_{c} \simeq 100 \mathrm{GeV}$, becomes $L_{\text {diff }}\left(T_{c}\right)=0.3 \mathrm{~cm}$. Taking again the flat spectrum for magnetic fields and assuming that their energy is $\sim T^{4}$ we obtain for the baryon number fluctuations at that scale $\delta\left(n_{B} / s\right) \sim 10^{-5} \gg 10^{-10}$. If magnetic fields are large enough, domains of matter and antimatter may exist at the scales 5 orders of magnitude larger than the neutron diffusion length. To our best knowledge, there were no studies of nonhomogeneous BBN with this type of initial conditions. It would be very interesting to see whether this may change BBN bounds on the baryon to photon ratio by changing the related predictions of the light element abundances. This possible analysis will not be attempted here.

A more conservative attitude is to derive bounds on the magnetic fields from the requirement that homogeneous BBN is not spoiled, i.e., $\Delta\left(L_{\text {diff }}, t_{c}\right)<n_{B} / s$ which becomes in terms of the parameters $(\xi$ and $\epsilon)$ defining the hypermagnetic background

$\log \xi$

$$
<\frac{\log \frac{\sigma_{c}}{T_{c}}-6.26+\frac{1}{2} \log \epsilon+14.88 \epsilon+\log \left[\Omega_{B} h_{100}^{2}\right]}{4-\epsilon} .
$$




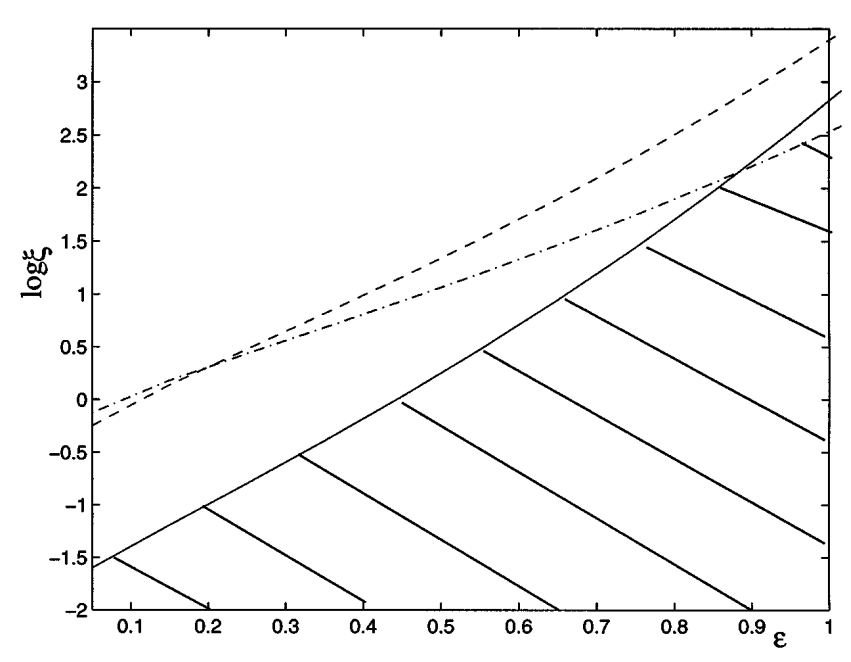

FIG. 1. We compare the bound (14) imposed by the absence of matter-antimatter domains in the standard BBN scenario (full line) with the constraint (15) imposed by the rate of universe expansion at the same epoch (dashed line). We also report for completeness the critical density bound (dot-dashed line). The bound given in Eq. (14) (shaded region) is more constraining for $\epsilon<1$ than the other two bounds. The plot is in the case $\Omega_{B} h_{100}^{2}=0.01, h_{100}=0.6$, and $\sigma_{c} / T_{c}=70$.

We plot it in Fig. 1 for a typical choice of the parameters and $0.05 \lesssim \epsilon \lessgtr 1$ (for $\sigma_{c} / T_{c} \simeq 70-100, \Omega_{B} h_{100}^{2} \simeq$ $0.1-0.01$, and $0.4<h_{100}<1$, this bound does not change significantly). Equation (14) represents a quite strong bound for blue spectra (i.e., $0<\epsilon<1$ ). For growing logarithmic spectra $[\epsilon>1$ in Eq. (13)] the fluctuations are parametrically smaller than $\left(n_{B} / s\right)$ at the neutron diffusion scale and then practically unconstrained by BBN. In order not to affect the Universe expansion at BBN it should hold (see, for instance, Kernan et al. in Ref. [3]) that $\rho_{H}<$ $0.27 \rho_{\nu}$ [where $\rho_{H}$ is the magnetic energy density defined after Eq. (12) and $\rho_{\nu}$ is the energy density contributed by the standard three light neutrinos for $T<1 \mathrm{MeV}]$. Therefore from Eqs. (9) and (12) we have

$$
\log \xi<\frac{\left(11.30-\frac{1}{2} \log \frac{\sigma_{c}}{T_{c}}\right) \epsilon+\log \epsilon-0.2}{4-\epsilon} .
$$

On the other hand, the critical density bound imposes that $\rho_{H}(t)<\frac{\pi^{2}}{30} N_{\text {eff }} T^{4}$ at any given time and, in particular, for $T=T_{c}$. These bounds are reported in Fig. 1. The bound of Eq. (14) turns out to be more stringent (by 2 orders of magnitude for logarithmic spectra with $\epsilon \ll 1$ ) than the bounds provided by the Universe expansion at the BBN time $[3,20]$.

We thank J. Cline, M. Joyce, H. Kurki-Suonio, and G. Veneziano for helpful discussions and comments.

[1] P. P. Kronberg, Rep. Prog. Phys. 57, 325 (1994).

[2] Y.B. Zeldovich, A. A. Rusmaikin, and D. D. Sokoloff, Magnetic Fields in Astrophysics (Gordon and Breach, New York, 1983).
[3] G. Greenstein, Nature (London) 223, 938 (1969); J. J. Matese and R. F. O'Connel, Astrophys. J. 160, 451 (1970); D. Grasso and H. Rubinstein, Phys. Lett. B 379, 73 (1996); B. Cheng et al., Phys. Rev. D 54, 4714 (1996); P. Kernan et al., Phys. Rev. D 54, 7207 (1996).

[4] G. Sigl, A. V. Olinto, and K. Jedamzik, Phys. Rev. D 55, 4582 (1997).

[5] T. Vachaspati, Phys. Lett. B 265, 258 (1991); K. Enqvist and P. Olesen, Phys. Lett. B 319, 178 (1993); T. W. Kibble and A. Vilenkin, Phys. Rev. D 52, 679 (1995); G. Baym, D. Bodeker, and L. McLerran, Phys. Rev. D 53, 662 (1996).

[6] M. Joyce and M. Shaposhnikov, Phys. Rev. Lett. 79, 1193 (1997).

[7] M. S. Turner and L. M. Widrow, Phys. Rev. D 37, 2743 (1988); B. Ratra, Astrophys. J. Lett. 391, L1 (1992); A. Dolgov and J. Silk, Phys. Rev. D 47, 3144 (1993).

[8] M. Gasperini, M. Giovannini, and G. Veneziano, Phys. Rev. Lett. 75, 3796 (1995); Phys. Rev. D 52, 6651 (1995); D. Lemoine and M. Lemoine, Phys. Rev. D 52, 1955 (1995).

[9] A. N. Redlich and L. C. R. Wijewardhana, Phys. Rev. Lett. 54, 970 (1985).

[10] M. Joyce, T. Prokopec, and N. Turok, Phys. Rev. D 53, 2930 (1996); G. Baym and H. Heiselberg, astro-ph/ 9704214.

[11] D. Biskamp, Nonlinear Magnetohydrodynamics, (Cambridge University Press, Cambridge, 1994).

[12] V.A. Kuzmin, V.A. Rubakov, and M.E. Shaposhnikov, Phys. Lett. 155B, 36 (1985).

[13] M. Giovaninni and M. Shaposhnikov, CERN-TH/97-264.

[14] M.E. Shaposhnikov, JETP Lett. 44, 465 (1986); Nucl. Phys. B287, 757 (1987).

[15] K. Kajantie, M. Laine, K. Rummukainen, and M. Shaposhnikov, Nucl. Phys. B495, 413 (1997).

[16] M. Carena, M. Quiros, and C. E. M. Wagner, Phys. Lett. B 380, 81 (1996); M. Laine, Nucl. Phys. B481, 43 (1996); J. M. Cline and K. Kainulainen, Nucl. Phys. B482, 73 (1996); M. Losada, hep-ph/9605266.

[17] J. Cline, K. Kainulainen, and K. Olive, Phys. Rev. Lett. 71, 2372 (1993); Phys. Rev. D 49, 6394 (1994).

[18] If the spectrum is dominated by parity noninvariant configurations then, in general, $\left\langle\delta\left(n_{B} / s\right)(\vec{x}, t)\right\rangle \neq 0$. Examples of these types of configurations can be found in [6]. If this is the case, the baryon asymmetry of the Universe can be a result of decay of Chern-Simons condensate $[13,14]$.

[19] J. Applegate, C.J. Hogan, and R. J. Scherrer, Phys. Rev. D 35, 1151 (1987); B. Banerjee and S. M. Chitre, Phys. Lett. B 258, 247 (1991); K. Jedamzik and G. M. Fuller, Astrophys. J. 423, 33 (1994); K. Jedamzik and G. M. Fuller, Astrophys. J. 452, 33 (1995); H. Kurki-Suonio, K. Jedamzik, and G. J. Mathews, astro-ph/9606011.

[20] Note also that, according to K. Jedamzik et al. (astro$\mathrm{ph} / 9606080$ ), the bound of (15) may, in fact, be absent, because there are other mechanisms, besides magnetic diffusivity, that can dilute the magnetic fields before the BBN. Then our bound remains the only one that can be applied to the small scale magnetic fields. 